\title{
New Analytical Developments for Uranium Based Powder
}

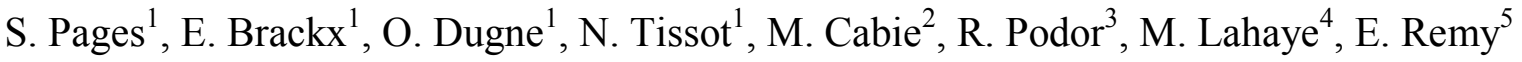 \\ 1. CEA, DEN, DTEC, SGCS, LMAC, 30207 Bagnols sur Cèze, France \\ 2. CP2M Université d'Aix Marseille III, Faculté des Sciences de Saint Jérôme, 13397 Marseille, France \\ 3. ICSM UMR 5257 - CEA / CNRS / UM2 / ENSCM, 30207 Bagnols sur Cèze, France \\ 4. Placamat, Université de Bordeaux 1, 33608 Pessac CEDEX, France \\ ${ }^{5 .}$ CEA, DEN, DRCP, SECA, LCAR, 30207 Bagnols sur Cèze,
}

Powders and divided solids are widely used in industry as intermediate or finished products in many fields: foods, cosmetics, construction, pharmaceuticals, transport, electronics and, of course, nuclear energy. Optimizing their use requires control of processing based on an understanding of the phenomena involved (sintering, chemical reactivity, purity, etc.). Modeling and understanding these phenomena require data and characteristics that may be difficult to obtain.

The present work cites examples illustrating different physicochemical characterization techniques suitable for analysis of uranium based powder samples (oxide, carbide, fluoride and metallic) in the raw state or after preparation (ion polishing) used in nuclear cycle.

The first section discusses dimensional characterization (particle size, morphology, porosity) by means of image analysis and preparation techniques: SEM, TEM, FIB, electron and X ray tomography with possible 2D and 3D image analysis. Morphological data in the form of the powder size and shape can be obtained with new developments in 2D analysis of processed SEM images [1]. Characterization of the pore size in a solid or a divided solid may cover a range of dimensions from $0.1 \mathrm{~nm}$ to $1 \mathrm{~cm}$. No characterization technique is capable of fully covering this measurement range, mainly due to the resolution of the measurement itself by the technique used. The full range is therefore generally covered several measurement techniques.

Two main types of techniques are used for the characterization of the pore structure: imaging and porosimetry techniques. The most commonly used invasive techniques are mercury porosimetry and the absorption isotherm technique; they can be used to measure pores of very small sizes (4 and $200 \mathrm{~nm}$, respectively) but cannot be applied to brittle samples because of high pressure constraints. Imaging techniques include optical microscopy, scanning electron microscopy (SEM), atomic force microscopy (AFM), and transmission electron microscopy (TEM). They are capable of determining the morphology of all open and closed pores, and of acquiring data on the surface porosity of the sample and its specific surface area. These techniques raise the problem of sample preparation, as the samples are observed on cross sections or thin sections that are mechanically polished, which may alter the pore morphology, especially with micrometer-size powder. In addition, the cross sections are made conductive for SEM or TEM observation by metallization of the entire surface, which can mask the presence of pores on the sample surface. A 3D interpretation of the 2D morphology observed by these imaging techniques is difficult due to parameters such as pore curvature and connectivity. The 3D morphology can be determined using additional techniques such as stereoscopy, confocal optical microscopy, X ray microtomography, MRI, focused ion beam (FIB) tomography, or coherent X ray diffraction microscopy. However, these techniques suffer from poorer spatial resolution, exceeding $50 \mathrm{~nm}$ except for FIB tomography, which has a spatial resolution of $10 \mathrm{~nm}$. 
Among the imaging techniques only image analysis by SEM, FIB tomography (Figure 1), and MRI are considered to be porosimetry techniques covering a wide range of pore sizes. FIB tomography also provides data on the powder specific surface area [2].

The second part of this work describes elemental chemical characterization. Low-voltage EDS spectroscopy on polished powder cross sections is used to map (Figure 2) the distribution of phases and to detect possible impurities in the solid grains and agglomerates. The contribution of this type characterization to understanding the reaction mechanisms of phase transformations (especially solid-gas reactions) is illustrated for the hydrofluorination of uranium dioxide. Sample preparation by mounting followed by ion polishing, optimization of the EDS analysis parameters, and the contribution to solid-gas reaction models are discussed in this study. Complementary surface analyses as Auger spectrometry allows powder surface oxidation.

The third section describes the characterization of the chemical reactivity of powders. These studies are performed by high-temperature in situ treatment in an environmental SEM, and allow observation of the powder morphology transformation.

The examples discussed concern materials used in nuclear fuel fabrication processes.

[1] E. Brackx, O. Dugne, B. Boichard, M. Bertrand, French Pattern FD 13531.

[2] H. Jaffel, Thèse Ecole Polytechnique City of Palaiseau France (2006).

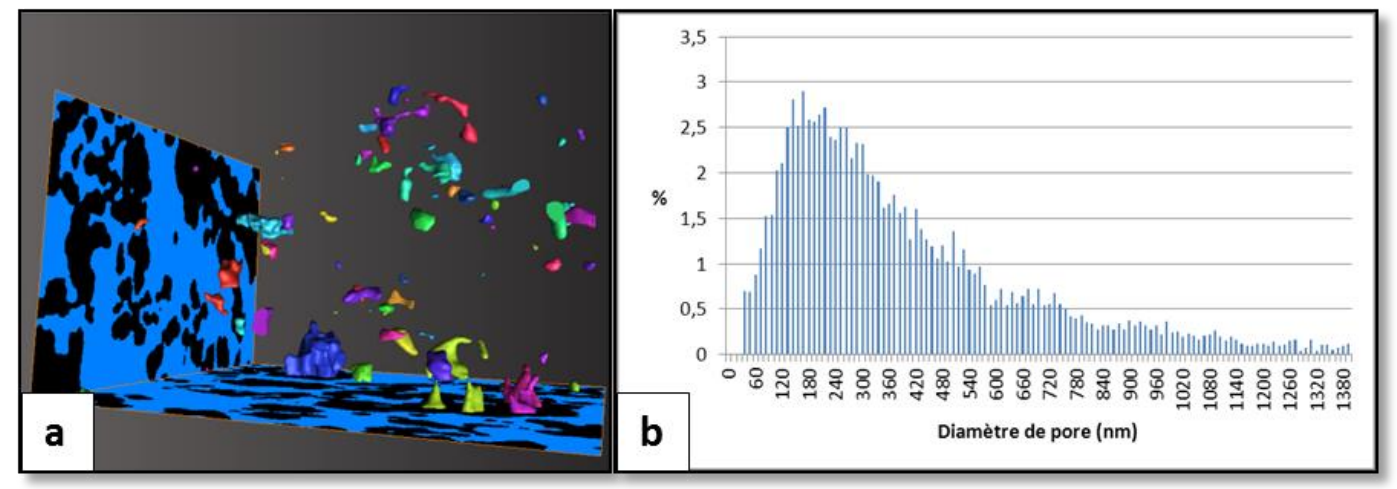

Figure 1. Illustration of the potential of FIB-SEM tomography for the characterization of porous media. Visualization of closed pores labeled in different colors (a), Pore size distribution determined after skeletonization of porous matrix.

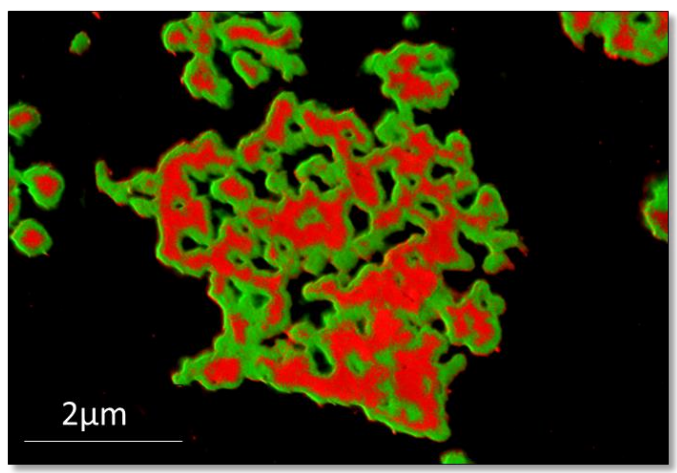

Figure 2. X-ray EDS mapping of $\mathrm{UO}_{2}$ powder partially transformed into $\mathrm{UF}_{4}$. Red and green phase represents respectively Oxygen and Fluor distribution. 